\title{
Fixed points of Lie group actions on surfaces
}

\author{
J. F. PLANTE \\ University of North Carolina, Chapel Hill, North Carolina 27514, USA
}

(Received 24 September 1984 and revised 23 May 1985)

\begin{abstract}
Let $G$ be a connected finite-dimensional Lie group and $M$ a compact surface. We investigate whether, for a given $G$ and $M$, every continuous action of $G$ on $M$ must have a fixed (stationary) point. It is shown that when $G$ is nilpotent and $M$ has non-zero Euler characteristic that every action of $G$ on $M$ must have a fixed point. On the other hand, it is shown that the non-abelian 2-dimensional Lie group (affine group of the line) acts without fixed points on every compact surface. These results make it possible to complete this investigation for Lie groups of dimension at most 3.
\end{abstract}

\section{0 . Introduction}

A classical result of Poincare [14] states that a vector field on a compact surface with non-zero Euler characteristic must be zero at some point. Another way to phrase this conclusion is to say that the flow tangent to the vector field must have a stationary point. This result was generalized to manifolds of arbitrary dimension by Hopf [6]. A flow is simply an action by the real line, so it is natural to consider the more general situation of Lie groups acting on manifolds.

Let $M$ be a smooth manifold and let $G$ be a connected finite dimensional Lie group. If $\Phi: G \times M \rightarrow M$ is a (left) action of $G$ on $M$ then for each $x \in M$ the set $G(x)=\{\Phi(g, x) \in M \mid g \in G\}$ is called the orbit of $x$ and the subgroup $G_{x}=$ $\{g \in M \mid \Phi(g, x)=x\}$ is called the stabilizer of $x . G_{x}$ is a closed subgroup of $G$ and the map $G / G_{x} \rightarrow G(x)$ defined by $g G_{x} \rightarrow \Phi(g, x)$ is continuous and bijective. Furthermore, the restriction of $\Phi$ to $G(x)$ is equivalent to the action of $G$ on $G / G_{x}$ by left translation. If $G_{x}=G$ then $G(x)=\{x\}$ and $x$ is called a stationary point or fixed point of the action. If every point is stationary then $G$ acts trivially. At the other extreme, if $G(x)=M$ for any (hence, all) $x \in M$ then the action is said to be transitive. The action is called effective if only the identity element of $G$ fixes every point of $M$. Given an arbitrary action by $G$ on $M$, the subgroup $H$ of $G$ which acts trivially is a closed normal subgroup and there is induced an effective action of the quotient Lie group $G / H$ on $M$.

Given a Lie group $G$ and a manifold $M$ we will say that the pair $(G, M)$ has the fixed point property if every continuous action of $G$ on $M$ has a fixed point. The result of Poincaré \& Hopf says that $(\mathbb{R}, M)$ has the fixed point property whenever $M$ is a compact manifold with non-zero Euler characteristic. In the present paper 
we will deal with the case in which $M$ is 2-dimensional, that is, we assume $M$ is a compact surface (possibly having a boundary). In our situation the orbits of the action must have dimension 0,1 , or 2 . The orbits of dimension zero are precisely the fixed points of the action. The 1-dimensional orbits are continuous images in $M$ of either the line or the circle. Even though $G(x)$ is not in general a smooth submanifold of $M$ it is the continuous image of the homogeneous space $G / G_{x}$ which has the structure of a real analytic manifold [11]. If every orbit has dimension one then the orbits are the leaves of a continuous foliation of $M$ [15], in which case $M$ has zero Euler characteristic [4], [9].

When an action of $G$ on $M$ is smooth then it is determined by the infinitesimal action on $M$ of the Lie algebra $g$ of $G$ [12]. If we deal with left actions then $g$ consists of all right invariant vector fields on $G$. In most cases it is easier to write down the infinitesimal action, that is, a Lie algebra homomorphism $g \rightarrow \mathfrak{X}(M)$ where $\mathfrak{X}(\boldsymbol{M})$ is the Lie algebra of smooth vector fields on $\boldsymbol{M}$.

$\$ 1$ contains some background results concerning the structure of actions on the one dimensional orbits which will be used frequently. In $\$ 2$ it is shown that the classical result of Poincare holds when $\mathbb{R}$ is replaced by a nilpotent Lie group. This generalizes the result by Lima [10] for the abelian case. $\$ 3$ gives constructions of actions without fixed points by the non-abelian 2-dimensional Lie group on every compact surface which illustrates how different the nilpotent and solvable cases are. $\S 4$ discusses the problem when $G$ is 3-dimensional.

\section{Orbits of dimension one}

If $G(x)$ is a one dimensional orbit of an action by a Lie group $G$ then the quotient space $G / G_{x}$ is diffeomorphic to the line or the circle. Since the action restricted to $G(x)$ is transitive we have an infinitesimal action $g \rightarrow \mathfrak{X}\left(G / G_{x}\right)$ with the property that for each point $p$ of $G / G_{x}$ there is a vector field $X$ in the image of $g$ such that $X(p) \neq 0$. It is a classical result, due to Lie in infinitesimal version [2], [3], that the only finite dimensional Lie groups which can act effectively and transitively on $\mathbb{R}$ are $\mathbb{R}$ (by translation), the affine group of the line (affinely), and $\overparen{\operatorname{SL}(2, \mathbb{R})}$ (the lifting to $\mathbb{R}$ of the linearly induced action on the circle of directions in $\mathbb{R}^{2}$ ). On $S^{1}$ only actions by $S^{1}$ (by rotations) and $\operatorname{SL}(2, \mathbb{R})$ (linearly induced allowing finite coverings) are possible. The infinitesimal form of these actions may be described as follows where $\partial / \partial x$ denotes the unit vector field on $\mathbb{R}$ : the image of the Lie algebra of $G=\mathbb{R}$, Aff $(\mathbb{R}), \widehat{\operatorname{SL}(2, \mathbb{R})}$ is generated by $\{\partial / \partial x\},\{\partial / \partial x, x \partial / \partial x\},\{(1+\cos x) \partial / \partial x,(\sin x) \partial / \partial x$, $(1-\cos x) \partial / \partial x\}$, respectively.

(1.1) Theorem. Suppose $\mathscr{L}$ is a finite dimensional Lie algebra of vector fields on $\mathbb{R}$ such that for each $p \in \mathbb{R}$ there is an $X \in \mathscr{L}$ such that $X(p) \neq 0$. Then $\mathscr{L}$ is isomorphic to one of the following Lie algebras:

(1) $\langle X\rangle$;

(2) $\langle X, Y \mid[X, Y]=X\rangle$;

(3) $\langle X, Y, Z \mid[X, Y]=X,[Y, Z]=Z,[X, Z]=2 Y\rangle$. 
Proof. If a vector field $X$ in a neighbourhood of $p$ has the form

$$
X=\left(a_{k}(x-p)^{k}+\cdots\right) \partial / \partial x
$$

where the $\cdots$ represents higher order terms and $a_{k} \neq 0$ then we say that $X$ has order $k$ at $p$. Computation shows that if $X$ and $Y$ have orders $i$ and $j$, respectively, at $p$ then $[X, Y]$ has order $\geq i+j-1$ at $p$. When $i \neq j,[X, Y]$ has order precisely $i+j-1$ at $p$. Let $\mathscr{L}_{p}^{k}=\{X \in \mathscr{L} \mid X$ has order $\geq k$ at $p\}$. The chain of subalgebras

$$
\mathscr{L}=\mathscr{L}_{p}^{0} \supset \mathscr{L}_{p}^{1} \supset \ldots
$$

must end with a last non-trivial subalgebra $\mathscr{L}_{p}^{l}$.

LEMMA. $\operatorname{dim}\left(\mathscr{L}_{p}^{k} / \mathscr{L}_{p}^{k+1}\right)=1(0 \leq k \leq l)$ and $\operatorname{dim} \mathscr{L}=l+1$. In particular, $l$ is independent of $p \in \mathbb{R}$.

Proof. Choose $X_{0} \in \mathscr{L}_{p}^{0}, X_{l} \in \mathscr{L}_{p}^{l}$. $\left[X_{0}, X_{l}\right]$ has order $l-1$ at $p,\left[X_{0},\left[X_{0}, X_{l}\right]\right]$ has order $l-2$ at $p$, etc. Let $X_{i-1}=\left[X_{0}, X_{i}\right], i=l, l-1, \ldots X_{i}$ has order $i$ at $p$ and it is easily checked that the $X_{i}$ 's are linearly independent and span $\mathscr{L}$. This proves the lemma.

An immediate consequence of the lemma is that $l \leq 2$ since $\left[X_{l-1}, X_{l}\right]$ has order $2 l-2$ at $p$, which implies $2 l-2 \leq l$. Therefore, $\mathscr{L}$ has dimension at most 3 . When $\mathscr{L}$ has dimension 1 it is spanned by a single vector field which never vanishes. When $\operatorname{dim} \mathscr{L}=2$ we choose $X_{0}, X_{1}$ as above to span $\mathscr{L}$. Since $\left[X_{0}, X_{1}\right]$ has order 0 at $p$

$$
\left[X_{0}, X_{1}\right]=a X_{0}+b X_{1}, \quad(a \neq 0) .
$$

Changing basis to $Y_{0}=X_{0}+(b / a) X_{1}, Y_{1}=(1 / a) X_{1}$ gives the relation $\left[Y_{0}, Y_{1}\right]=Y_{0}$. When $\operatorname{dim} \mathscr{L}=3, \mathscr{L}$ is spanned by $X_{0}, X_{1}, X_{2}$ with relations

$$
\begin{aligned}
& {\left[X_{0}, X_{1}\right]=X_{0}+a X_{1}+b X_{2} ;} \\
& {\left[X_{1}, X_{2}\right]=X_{2} ;} \\
& {\left[X_{0}, X_{2}\right]=2 X_{1}+c X_{2} .}
\end{aligned}
$$

Changing basis to $Y_{0}=X_{0}+a X_{1}+(b / 2) X_{2}, Y_{1}=X_{1}, Y_{2}=X_{2}$ and using the Jacobi identity we get the expected relations. This completes the proof of (1.1).

(1.2) THEOREM. If a finite dimensional Lie group acts smoothly effectively and transitively on $\mathbb{R}$ then its infinitesimal action is equivalent via a diffeomorphism to one of the following subalgebras of $\mathfrak{X}(\mathbb{R})$ :

(1) $\langle\partial / \partial x\rangle$;

(2) $\langle\partial / \partial x, x \partial / \partial x\rangle$;

(3) $\langle(1+\cos x) \partial / \partial x,(\sin x) \partial / \partial x,(1-\cos x) \partial / \partial x\rangle$.

Proof. We claim that in each of the cases of (1.1) there is a vector field in the Lie algebra which never vanishes. This is immediate from the lemma in case (1). The result in this case follows by taking flow-box coordinates which are defined for all of $\mathbb{R}$.

In case (2) we have the relation $[X, Y]=X$. We claim that $X$ never vanishes. Suppose $X(q)=0$ for some $q \in \mathbb{R}$. This means that $X \in \mathscr{L}_{q}^{1}$. If $Z \in \mathscr{L}$ is such that $Z(q) \neq 0$ then $[X, Z](q) \neq 0$, but this contradicts the fact that $[X, Z]$ must be a 
multiple of $X$. Since $X$ never vanishes we take coordinates for $\mathbb{R}$ so that $X=\partial / \partial x$. If $Y=f \partial / \partial x$ then the relation $[X, Y]=X$ becomes the differential equation $f^{\prime}=1$. Adjusting by a constant gives the Lie algebra in (2).

In case (3) it is helpful to consider a change of basis for the Lie algebra. Let

$$
U=\frac{1}{2}(X+Z) ; \quad V=Y ; \quad W=\frac{1}{2}(X-Z)
$$

The relations for the basis $\{U, V, W\}$ are

$$
[U, V]=W ; \quad[U, W]=-V ; \quad[V, W]=-U .
$$

We claim that $U$ never vanishes. Suppose $U(q)=0$ for some $q \in \mathbb{R}$. Since $\operatorname{dim}\left(\mathscr{L}_{q}^{0} / \mathscr{L}_{q}^{1}\right)=1$ there is a vector $V^{*}$ in the plane spanned by $V$ and $W$ such that $V^{*}(q)=0$. This implies that $\left[U, V^{*}\right](q)=0$. This is a contradiction since the set $\left\{U, V^{*},\left[U, V^{*}\right]\right\}$ is linearly independent which implies that one of these vector fields is non-zero at $q$. Now taking coordinates so that $U=\partial / \partial x, V=f \partial / \partial x, W=$ $g \partial / \partial x$ we obtain the system of equations $f^{\prime}=g, g^{\prime}=-f, g f^{\prime}-f g^{\prime}=1$ which, upon integration and adjustment of phase constant, yield

$$
V=(\sin x) \frac{\partial}{\partial x}, \quad W=(\cos x) \frac{\partial}{\partial x} .
$$

This completes the proof of (1.2).

Remark. These same arguments apply to the case of Lie groups acting on the circle. In this situation, case (2) (transitive action by the affine group of the line) is not possible. If such an action occurred it could be lifted to an action on the line equivalent to the standard affine action. However, the $Y$ vector field in this case on $\mathbb{R}$ has a unique zero which implies that it could not result from lifting a vector field on the circle.

\section{Actions by nilpotent groups}

Let $G$ be a simply connected nilpotent Lie group with Lie algebra $g$. If $M$ is a compact surface with Euler characteristic zero then $G$ can act without fixed points on $M$. To see this let $X \in g$ such that $X$ represents a non-zero element of $g /[g, g]$. Select a vector space basis for $g$ which includes $X$ as well as a basis for $[g, g]$. Define an infinitesimal action by sending $X$ to a nowhere-vanishing vector field on $M$ and sending all other basis elements to the identically zero vector field. When the Euler characteristic is non-zero it turns out that every action of $G$ on $M$ must have a fixed point. This was proved by Lima [10] for abelian $G$ and the proof of the nilpotent case follows similar lines.

(2.1) THEOREM. Every action by a connected nilpotent Lie group on a compact surface with non-zero Euler characteristic has a fixed point.

The proof of $(2.1)$ will require several preliminary results.

(2.2) LemMA. Let $\mathscr{C}$ be a disjoint collection of 2-sided embedded circles in a compact surface $M$. Then there exist finitely many circles $C_{1}, \ldots, C_{n}$ in $\mathscr{C}$ such that every circle in $\mathscr{C}-\left\{C_{1}, \ldots, C_{n}\right\}$ represents the trivial element of $H^{1}\left(M-\bigcup_{i=1}^{n} C_{i}\right)$. 
Remark. By '2-sided' we mean that the circle has a product neighbourhood homeomorphic to $S^{1} \times \mathbb{R}$ where the circle itself corresponds to $S^{1} \times\{0\}$. Each such circle determines an element of $H^{1}(M)$ by oriented transverse intersection number. The geometric meaning of the conclusion of (2.2) is that every circle distinct from $C_{1}, \ldots, C_{n}$ separates $\left(M-\bigcup_{i=1}^{n} C_{i}\right)$.

Proof of (2.2). The group $H^{1}(M)$ is finitely generated and free abelian and, therefore, so is the subgroup generated by the elements of $\mathscr{C}$. Let $C_{1}, \ldots, C_{n}$ be elements of $\mathscr{C}$ which generate this subgroup. If $C \notin\left\{C_{1}, \ldots, C_{n}\right\}$ were to separate $\left(M-\bigcup_{i=1}^{n} C_{i}\right)$ then the class represented by $C$ would not be in the subgroup generated by $\mathscr{C}$. This proves (2.2).

Now consider an arbitrary Lie group $G$ acting on the compact orientable surface $M$. By an exceptional minimal set of the action we will mean a nowhere dense (compact) minimal set consisting of uncountably many one dimensional orbits.

(2.3) LEMMA. The number of exceptional minimal sets in a compact surface $M$ is bounded by rank $H^{1}(M)$. In particular, it is finite.

Proof. Without loss of generality, assume that $M$ is connected. When the surface $M$ is orientable it follows from [13, (8.5)] that the number of exceptional minimal sets is bounded by $\frac{1}{2}$ rank $H^{1}(M)$. If $M$ is not orientable then we may lift the action to a 2 -fold orientable connected covering space $\tilde{M}$ of $M . \tilde{M}$ contains at least as many exceptional minimal sets as does $M$. Therefore, the number of exceptional minimal sets in $M$ is bounded by rank $H^{1}(M)$ when $\partial M=\varnothing$ and by (rank $H^{1}(M)-$ $\frac{1}{2}$ ) when $\partial M \neq \varnothing$. This completes the proof of $(2.3)$.

(2.4) Proposition. If a connected Lie group (not necessarily nilpotent) acts without fixed points on a compact surface $M$ then the union of all minimal sets of the action is itself a compact set.

Proof. Without loss of generality, assume that $M$ is connected. Since the action has no fixed points every orbit has dimension 1 or 2 . If some minimal set contains an open set then the minimal set is open and, therefore, all of $M$. Otherwise, all minimal sets are nowhere dense and consist of 1-dimensional orbits. By (2.3) the union of the exceptional minimal sets is compact. The only other type of nowhere dense minimal set is an embedded circle. Let $\mathscr{C}$ be the collection of all embedded circle orbits. The proof of (2.4) will be completed by showing that $\mathscr{C}$ is closed. Since the circles in $\mathscr{C}$ may not be 2 -sided in $M$ we consider instead (if necessary) the action lifted to a connected orientable 2-fold covering space of $M$. Assuming the elements of $\mathscr{C}$ are 2 -sided we apply (2.2) to obtain circles $C_{1}, \ldots, C_{n}$ such that every other element of $\mathscr{C}$ separates $\left(M-\bigcup_{i=1}^{n} C_{i}\right)$. Let $p_{k} \rightarrow q$ be a convergent sequence in $\left(M-\bigcup_{i=1}^{n} C_{i}\right)$ such that each $p_{k}$ is contained in a different element of $\mathscr{C}$. We claim that $q$ also lies on some circular orbit. Assume that the orbit of $q$ is not a circle. Since $q$ is not stationary (by hypothesis) there is, according to the proof of theorem (1.2), a one parameter subgroup of $G$ which determines a flow $\phi_{\mathrm{r}}$ on $M$ such that the $\phi_{t}$-orbit of $q$ coincides with $G(q)$. Note that for $k$ sufficiently large $p_{k}$ is moved by $\phi_{t}$. Since the action has no fixed points, the positive $\phi_{t}$ semi-orbit of $q$ passes 
twice through the same $\phi_{t}$ flow box. In particular, there exist points $r$ and $s$ on the $\phi_{t}$-orbit of $q$ such that $r$ and $s$ are endpoints of an arc which is transverse to $\phi_{t}$-orbits. On this arc there is a sequence of points $q_{k}$ such that each $q_{k}$ is on the same $\phi_{i}$-orbit as $p_{k}$ and the sequence $p_{k}$ converges either to $r$ or $s$ (depending on orientation). The simple closed curve in $\left(M-\bigcup_{i=1}^{n} C_{i}\right)$ obtained by taking the union of this transverse arc together with the segment of the $\phi_{t}$-orbit joining $r$ and $s$ meets each of the circular orbits $G\left(p_{k}\right)=G\left(q_{k}\right)$ with non-zero oriented intersection number, contradicting the fact that each $G\left(p_{k}\right)$ separates $\left(M-\bigcup_{i=1}^{n} C_{i}\right)$. Therefore, $G(q)$ must be in $\mathscr{C}$ and $\mathscr{C}$ must be a closed set. This proves (2.4).

Denote by $g$ the Lie algebra of the Lie group $G$. If $G$ acts on a manifold $M$ and $x \in M$, denote by $g_{x}$ the Lie subalgebra of $g$ corresponding to the closed subgroup $G_{x}$ of $G$. In general, $G_{x}$ is not normal (equivalently $g_{x}$ is not an ideal) but for a one dimensional orbit when $G$ is nilpotent it is.

(2.5) LEMMA. If $G(x)$ is a 1-dimensional orbit of an action by a connected nilpotent Lie group $G$ then $G_{x}$ is normal in $G$. Furthermore, if the action has no stationary points then $g_{y}=g_{x}$ for every $y$ in the closure of $G(x)$.

Proof. Restricting the action to $G(x)$ we are in the situation of a Lie group acting on $\mathbb{R}$ or $S^{1}$. Since $G$ is nilpotent, $g$ cannot have as quotient either of the cases (2), (3) of theorem (1.1). Therefore, $G$ acts on $G(x)$ in a manner equivalent to either translations of $\mathbb{R}$ or rotations of $S^{\mathbf{1}}$. Since the stabilizers of two points in the same orbit are conjugate $G_{x}$ must be normal in $G$. This proves (2.5) since $g_{x}$ varies semicontinuously with $x$.

Remarks. This argument also shows that for any finite dimensional nilpotent Lie algebra a codimension one subspace is a subalgebra iff it is an ideal. The argument in the proof of (2.5) also works for many groups which aren't nilpotent.

(2.6) LEMMA. If $\mathscr{L}$ is a nilpotent Lie algebra of dimension $\geq 2$ then the codimension one ideals of $\mathscr{L}$ are parametrized by a manifold of positive dimension.

Proof. When $\mathscr{L}$ is abelian of dimension $n$ then the ideals (subspaces) of codimension one are parametrized by $S^{n-1}$. When $\mathscr{L}$ is nilpotent of dimension $\geq 2$ then we claim $\operatorname{dim}(\mathscr{L} /[\mathscr{L}, \mathscr{L}]) \geq 2$. This in clearly true in dimension 2 since the only nilpotent Lie algebra in that case is actually abelian [7]. Assume the claim is true for nilpotent Lie algebras of dimension $n-1 \quad(n \geq 3)$. Take the quotient of an $n$-dimensional nilpotent Lie algebra by a one dimensional central ideal $\mathscr{J}$. Since $\mathscr{L} / \mathscr{J}$ has an abelian quotient of dimension $\geq 2$ so does $\mathscr{L}$, as claimed. It remains to observe that the codimension one ideals in $\mathscr{L}$ are in one-to-one correspondence with the codimension one subspaces of $\mathscr{L} /[\mathscr{L}, \mathscr{L}]$. If $V$ is a codimension one subspace of $\mathscr{L} /[\mathscr{L}, \mathscr{L}]$ then the preimage of $V$ in $\mathscr{L}$ under the quotient map is a codimension one ideal in $\mathscr{L}$. On the other hand, every codimension one ideal in $\mathscr{L}$ contains $[\mathscr{L}, \mathscr{L}]$ (since the only one dimensional Lie algebra is abelian) so its image has codimension one in $\mathscr{L} /[\mathscr{L}, \mathscr{L}]$. This proves $(2.6)$.

We are now ready to prove theorem (2.1). The proof will be by induction on the dimension of the nilpotent Lie group $G$ which we may assume to be simply 
connected. When $\operatorname{dim} G=1, G=\mathbb{R}$ and (2.1) is Poincaré's theorem. Assume now that $(2.1)$ is true whenever $\operatorname{dim} G \leq m-1, m \geq 2$. Suppose that a nilpotent group $G$ of dimension $m$ acts without fixed points on a compact surface $M$ such that $\chi(M) \neq 0$. The proof will be completed by showing that in such a case there would be a codimension one subgroup $G^{*}$ of $G$ and a 2 -dimensional submanifold $M^{*} \subset M$ such that:

(a) $\chi\left(M^{*}\right)=\chi(M)$

(b) $M^{*}$ is invariant under the action by $G$; and

(c) the restriction to $G^{*}$ of the action on $M^{*}$ has no fixed points.

Suppose the action of $G$ on $M$ consists of a single 2-dimensional orbit (transitive). The restriction of the action to any codimension one subgroup of $G$ (and not changing $\boldsymbol{M}$ ) satisfies (a), (b), (c). Assume now that the action has one-dimensional orbits. Let $g$ be the Lie algebra of $G$, and $N$ the manifold of codimension one ideals in $g$. Let $M_{1} \subset M$ be the subset consisting of one-dimensional orbits. According to (2.5) the continuous map $M_{1} \rightarrow N\left(x \mapsto g_{x}\right)$ is constant on orbit closures. Furthermore, its image is the same as that of its restriction to the union $M_{0}$ of minimal sets of the action (since every orbit has a minimal set in its closure). A circular orbit of the action is isolated if it has a neighbourhood in $M$ which does not meet any other circular orbits. The union of all circular orbits which are not isolated is a compact set $K$. By standard arguments using Poincaré maps every circular orbit contained in $K$ has a neighbourhood in $M_{0}$ (open in $M_{0}$ but not necessarily in $M$ ) which is either a cylinder or Moebius band bounded by circular orbits. Since $K$ is compact it is covered by finitely many such neighbourhoods $A_{1}, \ldots, A_{k}$. Let $M^{*}$ be the closure in $M$ of $\left(M-\bigcup_{i=1}^{k} A_{i}\right)$. (a) is satisfied since $\chi\left(A_{i}\right)=0, i=1, \ldots, k$. (b) is satisfied since each $A_{i}$ is invariant under the action by $G$. Since $M^{*}$ contains only isolated circular orbits it has only finitely many of them since $M^{*}$ is compact. According to (2.3) there are finitely many exceptional minimal sets and, therefore, $M^{*}$ contains finitely many minimal sets altogether. This means that the image of $\left(M_{1} \cap M^{*}\right)$ in $N$ is a finite set. Since $N$ is a manifold of positive dimension by (2.6) it follows that there is a codimension one ideal $g^{*} \subset g$ which is not equal to $g_{x}$ for $x$ in any one-dimensional orbit in $M^{*}$. Letting $G^{*}$ be the subgroup of $G$ corresponding to $g^{*}$ satisfies the requirement (c) above and the proof of theorem (2.1) is complete.

\section{Actions by the 2-dimensional non-abelian group}

There are two simply connected 2-dimensional Lie groups. In addition to the abelian group there is the (solvable) affine group of the line consisting of diffeomorphisms of the form $x \rightarrow a x+b(a>0)$. The Lie algebra of the affine group is isomorphic to the algebra generated by $X, Y$ with relation $[X, Y]=X$. If $M$ is any compact manifold with $\chi(M)=0$ we can define an action without fixed points by the affine group on $M$ by sending $X$ to the identically zero vector field and $Y$ to a nowhere vanishing vector field on $M$. So far this resembles the nilpotent case. In contrast to the nilpotent case, we have the following: 
(3.1) TheOREM. The 2-dimensional non-abelian Lie group acts without fixed points on every compact surface.

We begin our series of examples with a variation of an example given by Lima [10] of an action without fixed points on the disk.

The vector fields $X=\partial / \partial x, Y=x \partial / \partial x+y \partial / \partial y$ define an action without fixed points on the plane by the affine group. In order to construct an example on the disk we embed the plane as the interior of the disk. Since the boundary of the disk is to be a one-dimensional orbit it follows from the remark at the end of $\S 1$ that on the boundary we will need to require that $X \equiv 0$ and $Y \neq 0$ (at every boundary point). This is accomplished by shrinking and twisting the plane increasingly as we move away from the origin. Let $\lambda, \mu: \mathbb{R} \rightarrow \mathbb{R}$ be $C^{\infty}$ functions ( $\lambda$ increasing, $\mu$ decreasing) such that $\lambda(t)=0$ and $\mu(t)=1$ for $t \leq \frac{1}{2}, 0<\lambda(t), \mu(t)<1$ for $\frac{1}{2}<t<1$, $\lambda(t)=1$ and $\mu(t)=0$ for $t \geq 1$. If $(r, \theta)$ denotes polar coordinates define the vector field $\bar{Y}$ on the closed unit disk by

$$
\bar{Y}=\lambda(r) \frac{\partial}{\partial \theta}+r \mu(r) \frac{\partial}{\partial r} .
$$

We define a $C^{\infty}$ embedding $h$ of the plane onto the open unit disk as follows. Denote by $\phi_{t}$ and $\psi_{t}$ the $Y$ and $\bar{Y}$ flows, respectively. For any point $p$ in the plane we may choose a $t>0$ such that $\phi_{-t}(p)$ lies in the disk of radius $\frac{1}{2}$ centred at the origin. Define $h(p)=\psi_{t}\left(\phi_{-t}(p)\right)$. This definition does not depend on the value of $t$ chosen and $h$ is the identity map when $r \leq \frac{1}{2}$. Let $\bar{X}=h_{*}(X)$ on the interior of the unit disk. It is easily checked that $\bar{X}(p)$ approaches zero as $p$ approaches the unit circle so we extend $\bar{X}$ to the closed unit disk by letting it be identically zero on the unit circle. This gives an action on the closed disk without fixed points, by the affine group of the line.

It is worth noting that, in an example such as the foregoing, once the vector field $Y$ is chosen and the $X$ field is specified on certain subsets, $X$ is completely determined.

(3.2) LEMMA. Let $Y$ be the vector field in the plane given by $x \partial / \partial x+y \partial / \partial y$ and let $X$ be a second vector field defined on an annulus $x^{2}+y^{2} \geq C$ such that $[X, Y]=X$. Then $X$ is bounded and is determined uniquely by its values on any circle transverse to $Y$. If $X$ is defined on $\mathbb{R}^{2}$ then $X$ is a constant vector field.

Proof. Let $X=f \partial / \partial x+g \partial / \partial y$. The relation $[X, Y]=X$ is equivalent to the system of equations

$$
\begin{gathered}
x f_{x}+y f_{y}=0, \\
x g_{x}+y g_{y}=0,
\end{gathered}
$$

which is precisely the condition that the vector field $X$ is constant along orbits of the $Y$-flow (i.e. along radial lines). Since every orbit of the $Y$-flow tends to the origin as $t \rightarrow-\infty, X$ must be constant if it is defined at the origin. 
(3.3) Lemma. If $[X, Y]=X, Y=-x \partial / \partial x-y \partial / \partial y$, and $X$ is defined on the interior of the punctured disk $0<x^{2}+y^{2} \leq C$ then $\|X(x, y)\| \leq K\left(x^{2}+y^{2}\right)$ for some constant $K$.

Proof. The diffeomorphism of $\mathbb{R}^{2}-\{0,0\}$ to itself given in polar coordinates by $(r, \theta) \mapsto(1 / r, \theta)$ takes the vector field $(-x \partial / \partial x-y \partial / \partial y)$ to $(x \partial / \partial x+y \partial / \partial y)$. Applying lemma (3.2) we see that the vector field $X$ must be sent to a vector field of the form $k(\theta) \partial / \partial r+l(\theta) \partial / \partial t$ where $\partial / \partial r, \partial / \partial t(=(1 / r) \partial / \partial \theta)$ denote unit radial and tangential vector fields. This gives

$$
X=-r^{2} k(\theta) \frac{\partial}{\partial r}+r^{2} l(\theta) \frac{\partial}{\partial t}
$$

and (3.3) follows by taking $K=\sup _{\theta}\left[(k(\theta))^{2}+(l(\theta))^{2}\right]^{1 / 2}$.

(3.4) Lemma. Suppose $[X, Y]=X, Y=x \partial / \partial x-y \partial / \partial y$, and $X$ is defined on the open half plane $y>0$. If $X(0,1)$ has non-zero $x$-component then $X$ extends continuously to the closed half plane $y \geq 0$ so that the relation $[X, Y]=X$ remains satisfied and $X(x, 0)$ is a non-zero constant multiple of $\partial / \partial x$.

Proof. Let $X=f \partial / \partial x+g \partial / \partial y$. The condition that $[X, Y]=X$ is the system

$$
\begin{gathered}
x f_{x}-y f_{y}=0, \\
x g_{x}-y g_{y}=-2 g .
\end{gathered}
$$

The first equation says that $f$ is constant along orbits of the $Y$-flow. Let $p_{n}$ be a sequence of points in the open half plane which converges to a point $p$ on the $x$-axis. Since the phase portrait of $Y$ is 'saddle-like' there is a sequence $q_{n}$ of points in the open half plane which converges to a point on the positive $y$-axis and such that, for each $n, p_{n}$ and $q_{n}$ are on the same orbit of the $Y$-flow. Therefore, the $x$-component of $X$ extends continuously to the closed half plane (indeed, it is constant). It remains to show that the $y$-component of $X$ approaches zero as the $x$-axis is approached. The relation $[X, Y]=X$ says that the $Y$-flow takes one integral curve of the $X$-flow to another when the vector fields $X$ and $Y$ are transverse, as is the case at the point $(0,1)$. The $Y$-flow is normally hyperbolic (contracting) to the $x$-axis so the $X$-integral curves, thought of as graphs of functions of $x$, converge to zero in the $C^{r}$-topology provided $X$ is $C^{r-1}$ [5]. This proves (3.4).

We now describe constructions leading to the proof of theorem 3.1. Begin with a smooth vector field $Y$ on the 2 -sphere with a single source at which $Y$ is equivalent to $(x \partial / \partial x+y \partial / \partial y)$ in some local coordinates. Assume also that $Y$ has 2 saddle points such that $Y$ is equivalent to $(x \partial / \partial x-y \partial / \partial y)$ in a neighbourhood of each, and 3 sinks such that $Y$ is equivalent to $(-x \partial / \partial x-y \partial / \partial y)$ in a neighbourhood of each. We assume that all the zeros of $Y$ lie on the equator which is assumed to be invariant under the $Y$-flow. Also assume that $Y$ is invariant under reflection through the equator which we identify with the $x$-axis with the source as origin. A neighbourhood of the set consisting of the saddle points and sinks is shown in figure 1 . 


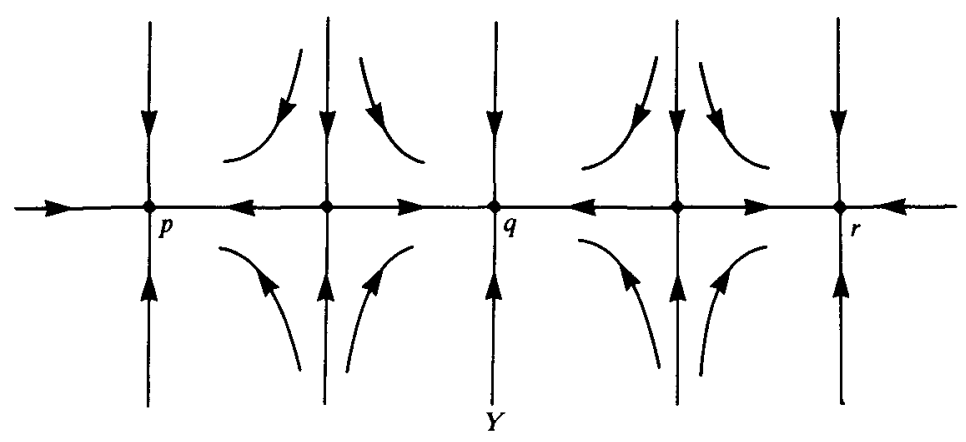

FIGURE 1

We wish to define a vector field $X$ on the sphere so that $[X, Y]=X$ and the zeros of $X$ coincide with the sinks of $Y$. ( $X$ will have index 1 at $p$ and $r$ and index 0 at $q)$. Removing the closed segment of the equator from $p$ to $r$ leaves an open set diffeomorphic to the plane via a diffeomorphism which takes $Y$ to the vector field $(x \partial / \partial x+y \partial / \partial y)$. Let $X$ be defined on this same open set to be the vector field corresponding to $\partial / \partial x$. Since $\partial / \partial x$ has no finite zeros in the sphere it has index 2 at $\infty$. We can arrange $X$ on the boundary of a neighbourhood of segment $[p, r]$ as indicated so that $X$ will be transverse to the stable manifolds of the saddle points.

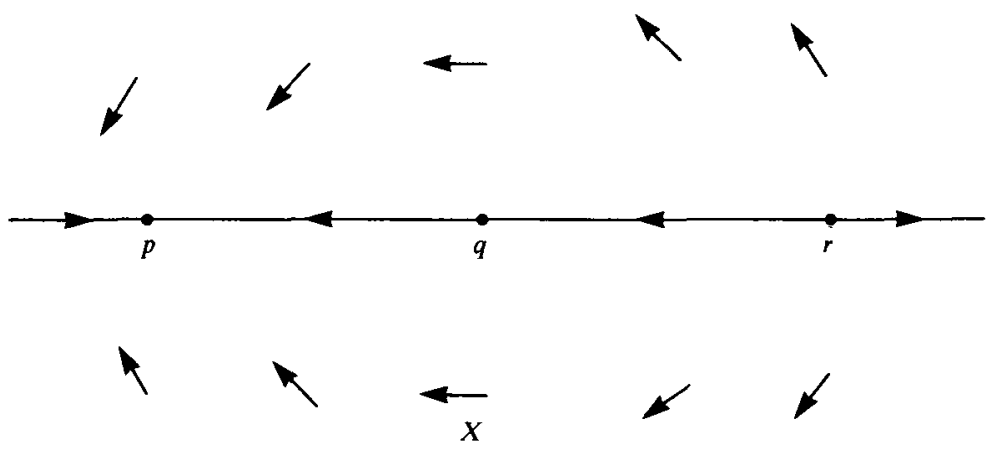

FIGURE 2

According to lemmas (3.3) and (3.4), $X$ extends to a continuous vector field on the sphere which vanishes only at the $Y$-sinks. Now restrict $X$ and $Y$ to the open subset $S^{2}-\{p, q, r\}$. For an open annulus around each of $p, q, r$ the vector field $Y$ is equivalent via a diffeomorphism to $x \partial / \partial x+y \partial / \partial y$ in an annulus $x^{2}+y^{2}>C$. Using an obvious modification of Lima's construction (used earlier to get an action of the affine group without fixed points on the disk) three times we obtain an action without fixed points of the affine group on a sphere with 3 open disks removed. Furthermore, $X \equiv 0$ on each boundary component and $Y$ never vanishes on the boundary. Since there are actions without fixed points of the affine group on the cylinder and the Moebius band $(X \equiv 0, Y$ never zero) we can obtain an action without fixed points 
on any compact surface by gluing together an appropriate number of disks, cylinders, Moebius bands, and 2-holed disks.

\section{Actions by 3-dimensional groups}

The results of the previous two sections tell whether a pair $(G, M)$ has the fixed point property when both $G$ and $M$ are 2-dimensional. In this section we show that the methods described earlier determine which simply connected 3-dimensional Lie groups can act without fixed points on which compact surfaces.

We begin by recalling the list of 3-dimensional Lie algebras ([7], [1]).

Abelian: $\langle X, Y, Z \mid[X, Y]=[X, Z]=[Y, Z]=0\rangle$

Nilpotent: $\langle X, Y, Z \mid[X, Y]=Z,[X, Z]=[Y, Z]=0\rangle$

Solvable: (including nilpotent) $\langle X, Y, Z|[X, Y]=0,[X, Z]=a X+b Y,[Y, Z]=$ $c X+d Y\rangle$

Simple: $\langle X, Y, Z \mid[X, Y]=Z,[Y, Z]=X,[Z, X]=Y\rangle$

$$
\langle X, Y, Z \mid[X, Y]=X,[Y, Z]=Z,[X, Z]=2 Y\rangle
$$

From $\S 2$ we know that the simply connected nilpotent and abelian groups act without fixed points on a compact surface $M$ iff $\chi(M)=0$. The situation for solvable groups which are not nilpotent hinges on whether the eigenvalues of ad $(Z)$ acting on the subspace spanned by $X$ and $Y$ are real. If ad $(Z)$ is diagonalizable we may select a new basis $\{U, V\}$, for the subspace spanned by $X$ and $Y$ so that

$$
[U, Z]=\alpha U, \quad[V, Z]=\beta V .
$$

Suppose, without loss of generality, that $\alpha \neq 0$. Letting $Z^{*}=(1 / \alpha) Z$ we have $\left[U, Z^{*}\right]=U$ so an action without fixed points on any compact surface can be defined by taking $U, Z^{*}$ to be, respectively, the vector fields $X, Y$ of $\S 3$ and $V \equiv 0$. If ad $(Z)$ has a real eigenvalue $\alpha$ ( $\neq 0$ assuming the algebra is not nilpotent) but is not diagonalizable then $U, V$ exist so that

$$
[U, V]=0, \quad[U, Z]=\alpha U+V, \quad[V, Z]=\alpha V .
$$

Again let $V \equiv 0, U, Z^{*}=(1 / \alpha) Z$ be as above to obtain an action without fixed points on any compact surface.

Now assume ad $(Z)$ has complex eigenvalues $\alpha \pm \beta i$. In this case $U$ and $V$ can be chosen so that

$$
[U, V]=0, \quad[U, Z]=\alpha U+\beta V, \quad[V, Z]=-\beta U+\alpha V .
$$

We claim that a simply connected Lie group with such a Lie algebra acts without fixed points on a compact surface $M$ iff $\chi(M) \geq 0$. Suppose $M$ admits an action without fixed points, that is, having only orbits of dimension 1 or 2 . If the action has a one dimensional orbit then $Z$ must be non-zero everywhere on the orbit. Suppose otherwise, namely that $Z(p)=0=W(p)$ where $W$ is in the plane spanned by $U$ and $V$. Since $W, Z,[W, Z]$ are linearly independent this would say that $p$ was fixed by the action, so we conclude that any zero of the vector field $Z$ must occur in a 2-dimensional orbit. Suppose $Z(p)=0$ and that $U(p)$ and $V(p)$ are linearly independent. In this case the above relations assert that the vector field $Z$ has index +1 at $p$. This says that the zeros of $Z$ are isolated and their sum is $\geq 0$ which implies $\chi(M) \geq 0$. On the other hand, such groups $d o$ act without fixed points on compact 
surfaces with $\chi \geq 0$. When $\chi=0$, let $Z$ be any nowhere vanishing vector field and $U \equiv 0 \equiv V$. When $M$ is the disk we can use a construction of the previous section. Define vector fields on the plane by

$$
U=\frac{\partial}{\partial x}, \quad V=\frac{\partial}{\partial y}, \quad Z=(\alpha x-\beta y) \frac{\partial}{\partial y}+(\beta x+\alpha y) \frac{\partial}{\partial y} .
$$

As in $\$ 3$ map the plane onto the open disk so that $U$ and $V$ approach zero at the boundary and $Z$ is non-vanishing and tangent to the boundary. Note that when $\alpha=0$ the $Z$-flow is periodic and the embedding $h$ used in the construction of $\S 3$ may be defined by $h(r, \theta)=(\nu(r), \theta)$ where $\nu$ is an increasing function such that $\nu(t)=t$ for $t \leq \frac{1}{2}$ and $\nu(t)=t /(1+t)$ for $t \geq 2$. This completes discussion of the solvable 3-dimensional groups.

The first of the simple Lie algebras listed is well-known as the Lie algebra of a compact simply connected group. This group is known to act transitively on $S^{2}$ and $P^{2}$ as a covering group of $\mathrm{SO}(3)$. It does not act without fixed points on any surface whose universal covering space is the plane. If it did there would be an action of the compact group on the plane which would imply (since the orbits are compact) that the plane has a 1-dimensional foliation with all leaves compact (which isn't the case). This argument works for any simply connected compact group.

Finally, the second Lie algebra listed is the Lie algebra of $\operatorname{SL}(2, \mathbb{R})$. SL $(2, \mathbb{R})$ acts without fixed points on the disk by Moebius transformations and consequently on $S^{2}$ by gluing together two disks. The adjoint representation induces actions without fixed points of $\operatorname{SL}(2, \mathbb{R})$ on $S^{2}$ and $P^{2}$. As observed in $\S 1, \operatorname{SL}(2, \mathbb{R})$ acts transitively on the circle and, therefore, acts without fixed points on the Moebius band, cylinder, Klein bottle and torus. We claim that the universal cover $\widehat{\operatorname{SL}(2, \mathbb{R})}$ cannot act without fixed points on any compact surface with $\chi<0$. As mentioned in $\S 1$ there is a basis $\{U, V, W\}$ of the Lie algebra for which the relations are

$$
[U, V]=W, \quad[U, W]=-V, \quad[V, W]=-U .
$$

In the proof of theorem (1.2) it was shown that the vector field $U$ never vanishes on a 1-dimensional orbit. If there is an action of $\widehat{\mathrm{SL}(2, \mathbb{R})}$ without fixed points on a compact surface $M$ then every zero of $U$ must occur in a 2-dimensional orbit. So, if $U(p)=0$ then $V(p)$ and $W(p)$ are linearly independent and the first two relations above imply that $U$ has index +1 at $p$ (rotation point). Since all zeros of $U$ have this property it follows that $\chi(M) \geq 0$.

\section{Concluding remarks}

The results in $\S \S 2,3$ show a sharp contrast between actions by nilpotent groups and actions by solvable (non nilpotent) groups. One consequence of $\S 3$ is that any Lie group which has the affine group of the line as quotient acts without fixed points on any compact surface. Such Lie groups have exponential growth. It is shown in [8] that a Lie group $G$ has exponential growth iff for some $X$ in the Lie algebra of $G$, ad $(X)$ has an eigenvalue with non-zero real part, and polynomial growth otherwise. In particular, $G$ has exponential growth iff its Lie algebra has a subalgebra isomorphic to one of the following: 
(i) $\langle X, Y \mid[X, Y]=X\rangle$;

(ii) $\langle X, Y, Z \mid[X, Y]=0,[X, Z]=X+\rho Y,[Y, Z]=-\rho X+Y(\rho \neq 0)\rangle$.

The first of these is the Lie algebra of the affine group. In the previous section it was shown that Lie groups having Lie algebras of type (ii) can act without fixed points on compact surfaces with $\chi \geq 0$ but cannot act without fixed points when $\chi<0$.

The results of this paper suggest that the following questions might have positive answers. In each case $G$ is a simply connected finite-dimensional Lie group and $\chi$ is the Euler characteristic of a compact surface.

(1) If $G$ has exponential growth does it act without fixed points on every compact surface with $\chi \geq 0$ ? What if $G$ is a solvable non nilpotent group?

(2) If $G$ is solvable and ad $(X)$ has a non-zero real eigenvalue for some $X$ in the Lie algebra of $G$ then does $G$ act without fixed points on every compact surface?

(3) If $G$ has polynomial growth does every action of $G$ on a compact surface with $\chi<0$ have a fixed point?

The questions in (1) have been answered affirmatively when $G$ has dimension $\leq 3$. A group having polynomial growth is a compact extension of a solvable group having polynomial growth [8]. As pointed out in the last section compact groups always have a fixed point when $\chi \leq 0$. Since a fixed point for the action by the solvable subgroup would result in a compact orbit (with finite fundamental group) of an action of $G$ on the universal cover space of $M$, question (3) reduces to a question about actions by solvable groups. Furthermore, the answer to (3) is affirmative for nilpotent groups, which have polynomial growth.

\section{REFERENCES}

[1] L. Auslander, L. Green \& F. Hahn. Flows on Homogeneous Spaces. Annals of Math. Studies \#53. Princeton U. Press, 1963.

[2] R. Bryant. Lie's theorem on group actions on one dimensional manifolds. Seminar notes, U. of North Carolina.

[3] J. Campbell. Introductory Treatise on Lie's Theory of Finite Continuous Transformation Groups. Chelsea: New York, 1966.

[4] G. Hector \& U. Hirsch. Introduction to the Geometry of Foliations, Part A. Vieweg: Braunschweig, 1981.

[5] M. Hirsch, C. Pugh \& M. Shub. Invariant Manifolds. Lecture Notes in Math. \#583. Springer Verlag: Berlin/Heidelberg, 1977.

[6] H. Hopf. Vektorfelder in n-dimensionaler Mannigfaltigkeiten. Math. Ann. 96 (1927), 225-250.

[7] N. Jacobson. Lie Algebras. Dover: New York, 1979.

[8] J. Jenkins. Growth of connected locally compact groups. J. Funct. Anal. 12 (1973), 113-127.

[9] H. Kneser. Reguläre Kurvensharen auf Ringflächen. Math. Ann. 19 (1924), 135-154.

[10] E. Lima. Common singularities of commuting vector fields on 2-manifolds. Comm. Math. Helv. 39 (1964), 97-110.

[11] D. Montgomery \& L. Zippin. Topological Transformation Groups. Interscience: New York, 1955.

[12] R. Palais. A Global Formulation of the Lie Theory of Transformation Groups. Mem. Amer. Math. Soc. \#22. Providence, 1957.

[13] J. Plante. Foliations with measure preserving holonomy. Annals of Math. 102 (1975), 327-361.

[14] H. Poincaré. Sur les courbes définies par une équation différentielle. J. Math. Pures Appl. (4) 1 (1885), 167-244.

[15] H. Whitney. Regular families of curves. Annals of math. 34 (1933), 244-270. 